\title{
X-ray Quantitative Microanalysis Maps across Interfaces of a Cu-Al Roll Bonded Laminate with an Annular Silicon Drift Detector
}

\author{
Hendrix Demers ${ }^{1}$, Nicolas Brodusch ${ }^{1}$, Richard Wuhrer ${ }^{2}$, Ken Moran ${ }^{3}$, Patrick Woo ${ }^{4}$ and Raynald \\ Gauvin $^{1}$ \\ 1. Department of Mining and Materials Engineering, McGill University, Montreal, Quebec, Canada. \\ 2. Advanced Materials Characterisation Facility, University of Western, Sydney, NSW, Australia. \\ ${ }^{3 .}$ Moran Scientific Pty Ltd, Bungonia, NSW, Australia. \\ ${ }^{4 .}$ Hitachi High-Technologies Canada Inc., Toronto, Canada.
}

The scanning electron microscope (SEM) was primary developed for imaging applications. With the introduction of the $\mathrm{Si}(\mathrm{Li})$ energy dispersive spectrometer (EDS), simultaneous imaging and x-ray microanalysis became possible. However, long working distance and high current were needed because the position and small solid angle of the EDS detector. SEM was initially and is still optimized for imaging applications, where the high spatial resolution is generally obtained at short working distance. This problem is still relevant today and unfortunately $\mathrm{x}$-ray microanalysis is never performed in the best imaging conditions, i.e., not with the smallest probe size. With the introduction of an annular silicon drift detector (SDD) system, scanning electron microscopy is facing a revolution. This detector is inserted below the objective lens and has four segments which give a higher solid angle (up to $1.2 \mathrm{sr}$ ). Also, a lower working distance and probe current can be used. An improved spatial resolution becomes possible during x-ray microanalysis. However, the effect of the detector geometry and position on the quantification microanalysis is unknown.

Because of the position of the detector, Mylar windows are used to prevent the backscattered electrons (BSEs) to damage the SDD segments. Three window thicknesses are available for this detector and their effect on the x-ray spectra is shown in Figure 1. The shape of the background was strongly affected by the window absorption at low x-ray energy. For accurate quantitative analysis, the calculation of peak net intensity depends on the background subtraction method used. Different approaches are currently studied with this annular SDD. Another artefact created by the window is the generation of $\mathrm{C}$ and $\mathrm{O}$ peaks and bremsstrahlung $\mathrm{x}$-rays in the window by the BSEs. Figure 2 shows the variation of the output count rate and the intensity of the $\mathrm{Cu} \mathrm{L} \mathrm{L}_{\alpha}$ peak with the working distance for the three window thicknesses. An optimum working distance was observed for the $\mathrm{Cu} \mathrm{L}_{\alpha}$ peak as predicted by the calculation of the solid angle of this detector [1-2]. However, no decrease of the output count rate was observed. The x-ray emission in the window negates the effect of the solid angle. This effect is more pronounce at high accelerating voltage. Recently, abnormal ZAF results were observed across interfaces of a $\mathrm{Cu}-\mathrm{Al}$ roll bonded laminate with conventional detectors (WDS and EDS) geometry [3]. This effect was not observed in the preliminary results obtained with the annular SDD as shown in Figure 3, where spectra from each of the four segments were compared.

The effect of this detector geometry and position on the correction model is currently studied. With adapted correction model, the annular SDD with is larger solid angle will clearly revolution the quantification microanalysis by moving from point analysis to quantitative micrograph with simultaneous electron imaging. Also, since the count rate can be as high as 1,500 kcps with our system, which lower significantly the detection limit of elements as well the minimum feature sizes of different phases that can be distinguished. 


\section{References:}

[1] N. J. Zaluzec, Microscopy Today 17 (2009), pp. 56-59.

[2] H. Demers, et al, Microscopy and microanalysis 19 (2013), pp. 364-365.

[3] H. Demers, et al, Microscopy and microanalysis 19 (2013), pp. 1250-1251.
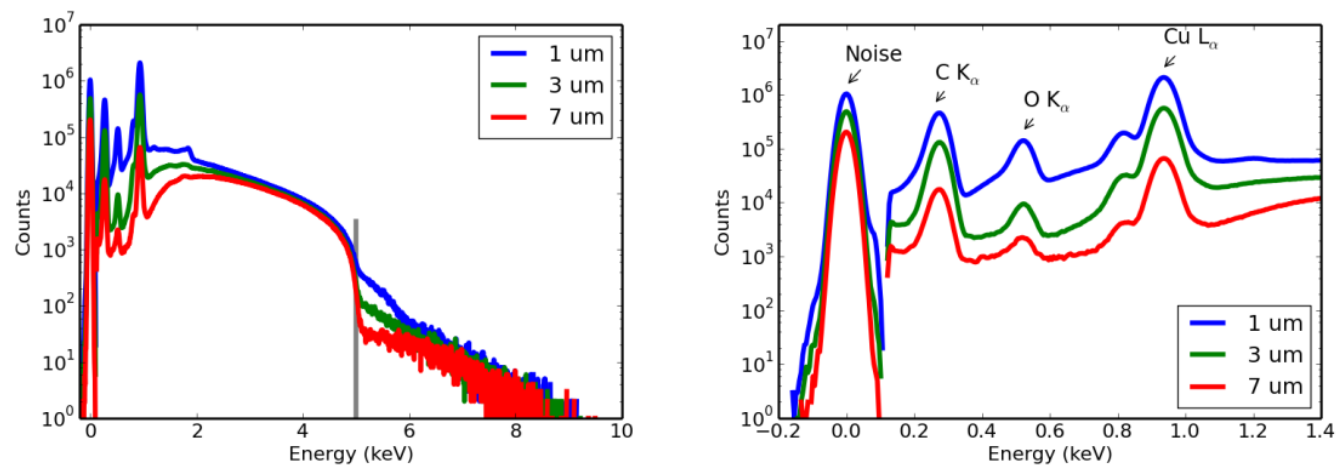

Figure 1. Copper sample spectra with three different window thicknesses for annular silicon drift detector (SDD). The gray line shows the expected Duane-Hunt limit at $5 \mathrm{keV}$. The $\mathrm{C} \mathrm{L} \alpha$ peak intensity decrease by 3.2 times with the $3 \mu \mathrm{m}$-thick window and 30 times with the $7 \mu \mathrm{m}$-thick window.
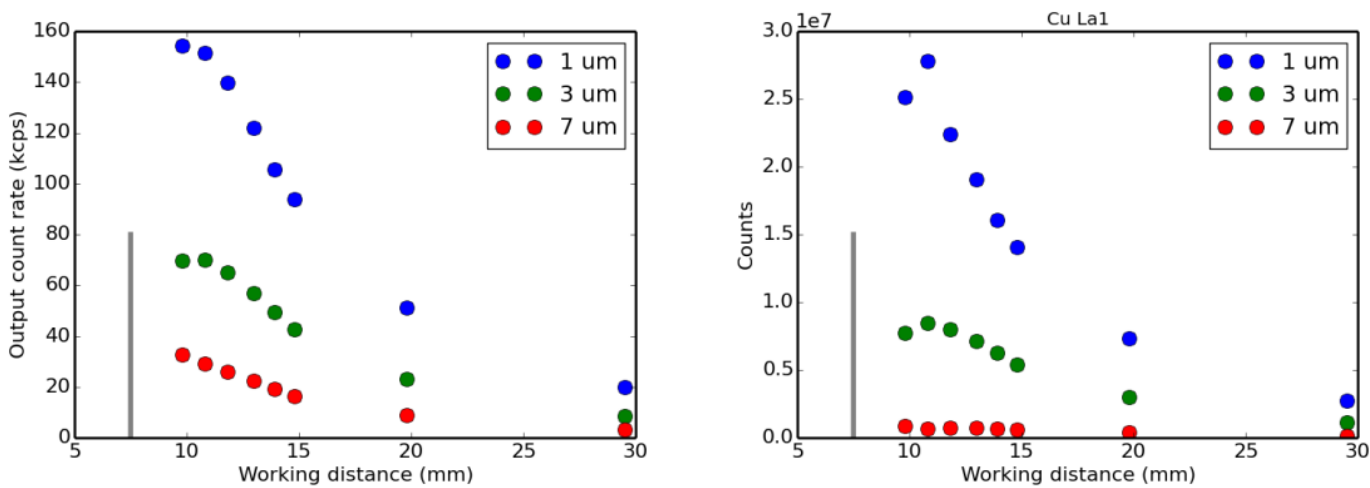

Figure 2. Variation of the experimental output count rate with working distance for three different window thicknesses for annular silicon drift detector (SDD). The gray line shows the detector bottom position at $7.5 \mathrm{~mm}$.
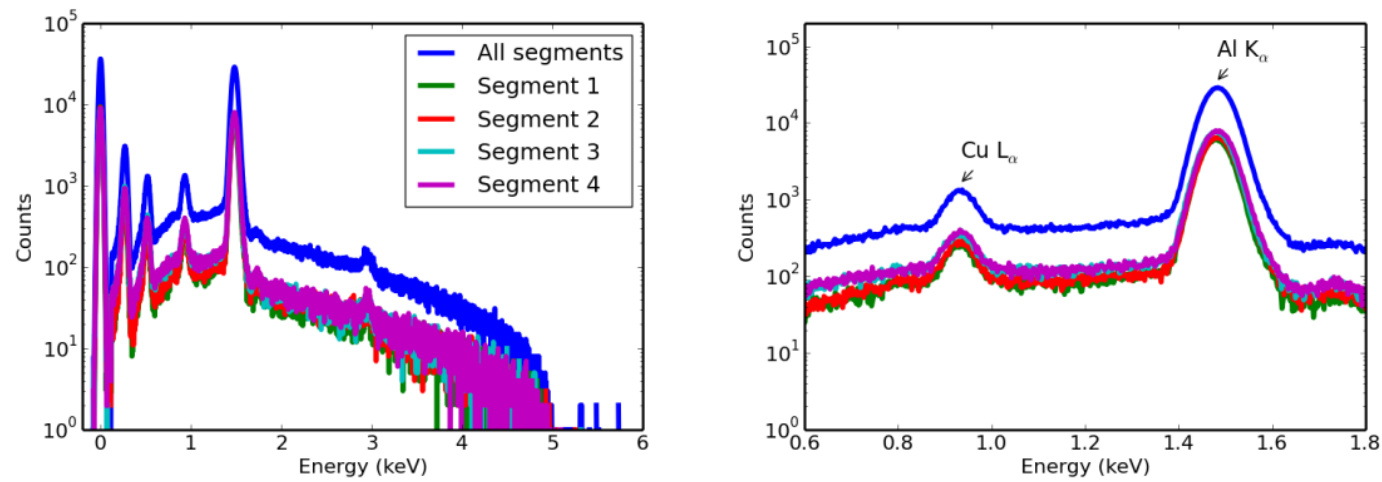

Figure 3. Experimental spectra comparison of the four segments of an annular silicon drift detector (SDD). Each spectrum were obtained with an acquisition time of $30 \mathrm{~s}$. However, the intensity different between segment spectra was probably caused by a change of the probe current between measurement. 